\title{
SAR86: streamlined for success
}

A recent ISME Journal paper presents a genomic analysis of the abundant marine gammaproteobacterial clade SAR86, revealing metabolic details that shed light on the role of these organisms in the ocean.

The SAR86 clade is ubiquitous and highly abundant in surface ocean samples, but until recently the only genomic data available to researchers comprised glimpses of the genome sequence from metagenomic samples, and, like the majority of the bacteria and archaea in the ocean, the clade remains uncultivated. In an approach that is likely to become more common in the future, Chris Dupont and colleagues assembled the genome sequence data for SAR86 by combining metagenomic analysis with single-cell genomics. They used metagenomic data from the Sorcerer II Global Ocean Sampling data set alongside single-cell genome reconstruction from seawater samples to generate four partial SAR86

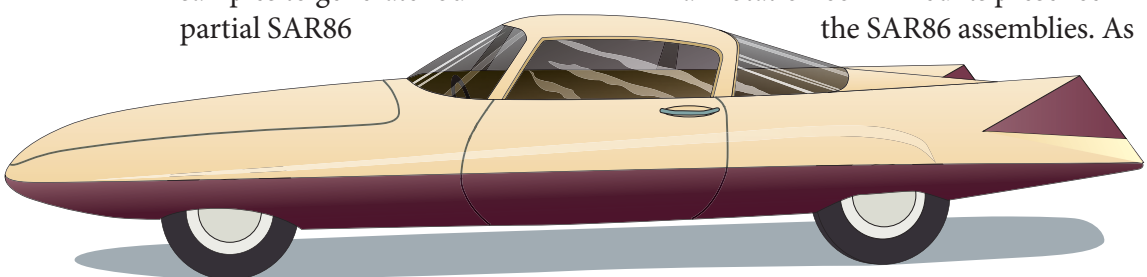

genome assemblies. Analyses of the overall gene content and organization confirmed that the assemblies were representative of the SAR86 genomes present in natural populations.

The SAR86 genome assemblies range from 1.25 to $1.7 \mathrm{Mb}$ in size and exhibit low GC content. Detailed analysis revealed evidence of metabolic streamlining, with the absence of pathways required for the biosynthesis of key vitamins and cofactors and the amino acids histidine, methionine and arginine. The authors also noted a surfeit of putative TonB-dependent outermembrane receptors in several genomic regions, many of which contained genes encoding enzymes involved in lipid degradation, perhaps indicating a particular focus on the uptake and degradation of lipids. SAR86 was the 16 S ribosomal RNA clade in which proteorhodopsin was first identified, and the genome annotation confirmed its presence in the SAR86 assemblies. As autotrophic carbon fixation pathways are absent, the authors suggest that these organisms are aerobic chemoheterotrophs that use proteorhodopsin to generate ATP. Proteorhodopsin requires the pigment retinol for functionality but, interestingly, the retinol biosynthesis pathway was not detected in the genome analysis, suggesting that SAR86 scavenges exogenous retinol or uses a structurally related pigment.

The metabolic streamlining present in SAR86 echoes that observed in the alphaproteobacterial clade SAR11, another extremely abundant member of the surface marine picoplankton. There are, however, sufficient differences in the metabolic capabilities of these two highly successful lineages to indicate that they may avoid competing for the same carbon sources. Representatives of the SAR11 clade have been successfully isolated in culture; the genomic information revealed in this paper may allow researchers to achieve the same goal for SAR86.

Sheilagh Molloy

ORIGINAL RESEARCH PAPER Dupont, C. L. et al. Genomic insights to SAR86, an abundant and uncultivated marine bacterial lineage. ISME J. 15 Dec 2011 (doi: 10.1038/ismej.2011.189) 